\title{
Transmission Power Control for Wireless Sensor Network
}

\author{
Kuo-Hsien Hsia \\ Department of Electrical Engineering, Far East University \\ No. 49, Zhonghua Road, Xinshi, \\ Tainan 74448, Taiwan (R.O.C.) \\ Chung-Wen Hung ${ }^{\dagger}$, Hsuan T. Chang, Yuan-Hao Lai \\ Department of Electrical Engineering, National Yunlin University of Science \& Technology \\ No. 123, Section 3, University Road, \\ Douliou, Yunlin, 64002, Taiwan (R.O.C.) \\ E-mail:khhsia@cc.feu.edu.tw, \{广'wenhung; htchang,m10312040\}@yuntech.edu.tw \\ www.yuntech.edu.tw
}

\begin{abstract}
Wireless sensor networks can be widely applied for a security system or a smart home system. Since some of the wireless remote sensor nodes may be powered by energy storage devices such as batteries, it is a very important issue to transmit signals at lower power with the consideration of the communication effectiveness. In this paper, we will provide a fuzzy controller with two inputs and one output for received signal strength indicator (RSSI) and link quality indicator (LQI) to adjust transmission power suitably in order to maintaining a certain communication level with a reduced energy consumption. And we will divide the sampling period of a sensor node into four intervals so that the sensor node radio device does not in receiving or transmission status all the time. Hence the sensor node can adjust transmission power automatically and reduce sensor node power consumption. Experimental results show that the battery life can be extended to about 10 times for the designed sensor node comparing to a normal node.
\end{abstract}

Keywords: Transmission power control, fuzzy control, wireless sensor network, internet of thing.

\section{Introduction}

Sensors play an important role in a variety of intelligent applications or control systems. If a sensor cannot transmit the sensed message to the control panel, the control panel will never know what control signal to send. Thus one may say "No sensor, no control." As the development of wireless communication, a sensor module, which containing sensor unit and wireless transmission unit, can transmit the sensed information to the information center in wireless way. Such sensing modules can be distributed in different locations to form a wireless sensor network and transmit the sensed information back to the control center. Wireless sensor networks become the key technology for the IoT (internet of things) [1]. Since some of the wireless remote sensor nodes may be powered by energy-storage devices such as batteries, it is a very important issue to transmit signals at lower power with the consideration of the communication effectiveness. Researchers used TPC (transmission power control) to achieve the goal of maintaining communication quality with reduced node power consumption. Ikram et al used SINR (signal to interference plus noise ratio) and SNR (signal to noise ratio) to determine a golden receive power range of RSSI (received signal strength indicator) to ensure the success of signal transmission [2]. Ramakrishnan and Krishna developed a fuzzy logic based TPC for the consideration of energy efficiency [3]. However, only RSSI cannot guarantee a good communication in an environment with interference signals. Kim et al used 
LQI (link quality indicator), which is determined by signal strength and detected SNR, to determine if there is interference in the environment [4]. PER (packet error rate) is usually used as a performance index in determining an acceptable range of wireless signal transmission. Many documents showed that a system with PER over 1\% may not retrieve the complete transmitted data [5-7]. So we set the allowable PER to be within $1 \%$ in our research.

\section{Transmission Power Control}

Maximizing the transmission power of each node in a wireless sensor network system can minimize the PER and make the transmission distance to the farthest. But this will lead to unnecessary power consumption and shorten the battery life of a wireless sensor node. Hence to control the transmission power with a certain level of communication being guaranteed is an important topic of transmit power control.

In general, the steps for controlling transmission power is as follows. At first the information about the transmission status, such as RSSI, LQI or SNR, of the node is gathered. Based on the transmission state information, the transmission power of the node is judged whether in an appropriate range or not. This range is determined by a large of experiments. If it is out of the range, then the transmission power has to be adjusted for successful communication.

The architecture of the TPC is shown in Fig. 1. It is implemented by TI CC430F6137 development board in this study. A Sub- $1 \mathrm{GHz}$ transmission packet with the information about RSSI, LQI and CRC checking results attached will be received by the development board. RSSI, in $\mathrm{dBm}$, can be used for determining whether the node transmission power is appropriately set or not. LQI represents the quality of connection in the current environment. In CC430F6137, LQI is expressed as a 7bit positive integer. The lower the value is, the better the communication quality is. In this study, LQI will be used as a supplement. If the communication quality is too bad, then the transmission power will be added immediately to reduce the PER.

For CC430F6137, the transmission power of the Sub$1 \mathrm{GHz}$ is in the range of $10.7 \mathrm{dBm}$ to $-69.2 \mathrm{dBm}$. A total of 121 transmission power parameters can be set. To

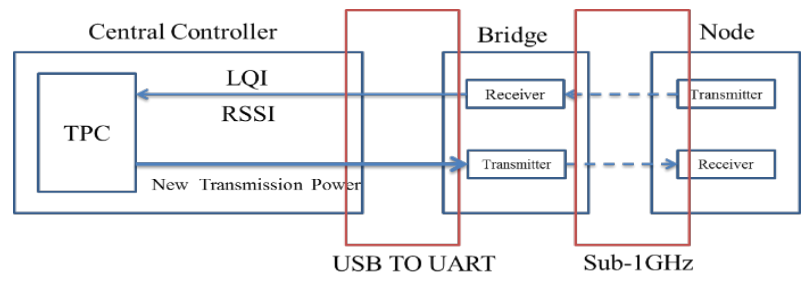

Fig. 1. Architecture of transmission power control.

prevent large variation of the parameters, only the transmission powers in the range of $10 \mathrm{dBm}$ to $-34 \mathrm{dBm}$ are used. There are 45 emission power parameters with intervals about $1 \mathrm{dBm}$ in this range.

The transmission power is determined by the central controller with the RSSI and LQI at the end of a packet and then transferred to the nodes via bridge to adjust the transmission power of the nodes.

\section{Fuzzy Transmission Power Controller}

In order to establish the RSSI standard receiving range and the LQI threshold, a large number of experiments are needed to find out the relationship between RSSI/LQI and PER. The scenario of the experiments are described as follows:

1. There are a central controller, a bridge, and a node in our experiment. The central controller can command the node to transmitting data with a designated transmission power.

2. The initial emission power is set to $0 \mathrm{dBm}$. This is the default set of CC430F6137. The distance between the node and the bridge (N-B distance) is $12 \mathrm{~m}$.

3. Since the RSSI value is easily disturbed by the environment and this may cause the output of the central controller variated frequently, a Kalman filter is used to eliminate the RSSI disturbance.

For the issue of RSSI, the central controller command the node to transmit a 32-byte data and extract the information of RSSI, LQI, and CRC checking result. The N-B distance varies from $1 \mathrm{~m}$ to $12 \mathrm{~m}$ stepping by $1 \mathrm{~m}$. At each distance, the node transmits 1000 data to the central controller through the bridge. The PER is defined by (1). And the resulting relationship of PER and RSSI is shown in Fig. 2.

$$
\mathrm{PER}=(\#(\text { lost package })+\#(\text { error package })) / 1000
$$


For the issue of LQI, an extra node transmitting noise is added to the experiments for RSSI. The N-B distance varies from $1 \mathrm{~m}$ to $12 \mathrm{~m}$ stepping by $1 \mathrm{~m}$. At each distance, the node transmits 1000 data to the central controller through the bridge in the noised environment. The PER is also defined by (1). The resulting relationship of PER and RSSI is shown in Fig. 3.

Refer to Fig. 2, the PER at RSSI equaling $-81 \mathrm{dBm}$ is $1.1 \%$. Hence we define the PER value as the lower threshold. Fig. 4 shows the RSSI of 500 data transmitted in $0 \mathrm{dBm}$. The drift range is $9 \mathrm{dBm}$. Hence the higher threshold can be set as $-72 \mathrm{dBm}$. The LOI threshold is set as 47 since the PER rising rapidly when LQI over 47 in Fig. 3. If the RSSI and LQI are in these ranges, the transmission power is maintained. If any one of them is out of the range, the transmission power will be adjusted by the fuzzy transmission power controller.

The membership functions of RSSI and LQI are defined in Figs. 5 and 6. The output of the controller, PAP, is an adjustment parameter. 7 fuzzy numbers of PAP are set for the controller. For the case that no power adjustment is needed, the output is $\mathrm{AZ}$ which is set as $0 \mathrm{dBm}$. To set appropriate PAPs for the controller, we design an experiment to get the adjustment amount of node transmission power to tune the RSSI to $-72 \mathrm{dBm}$ when RSSI is $-100,-94$, and $-81 \mathrm{dBm}$. The experimental result is shown in Fig. 7. The values marked in red are intervals from PL, PM, PS to high threshold. So we set the cores of PL, PM, and PS as 30,22 , and $7 \mathrm{dBm}$, respectively. The cores of NL, NM, and NS are obtained in a similar way. The resulting membership functions are defined in Fig. 8. The control laws are as follows:

IF (LQI is PM) or (RSSI is NL) then (PAP is PL).

IF (LQI is PS) and (RSSI is not NL) then (PAP is PM). IF (LQI is AZ) and (RSSI is not NM) then (PAP is PM).

IF (LQI is AZ) and (RSSI is not NS) then (PAP is PS).

IF (LQI is AZ) and (RSSI is not AZ) then (PAP is AZ).

IF (LQI is AZ) and (RSSI is not PS) then (PAP is NS).

IF (LQI is AZ) and (RSSI is not PM) then (PAP is NM).

IF (LQI is AZ) and (RSSI is not PL) then (PAP is NL). Experiments show that the designed fuzzy controller can control the transmission power efficiently, and the PER can be reduced to under $1 \%$. However, the

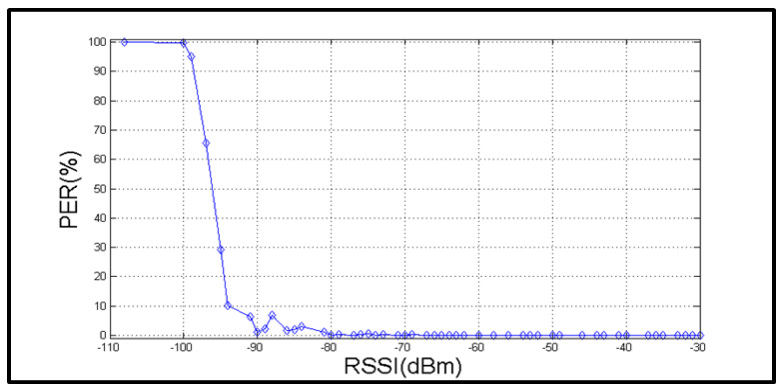

Fig. 2. Relationship of PER and RSSI.

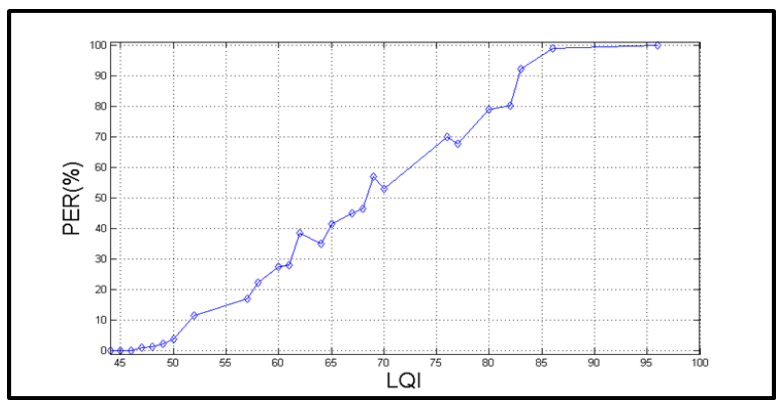

Fig. 3. Relationship of PER and LQI.

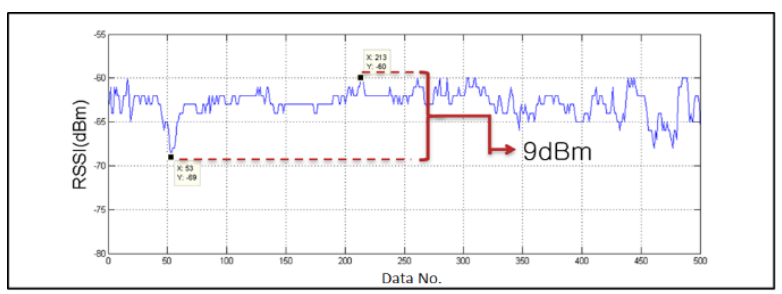

Fig. 4. Drift range of RSSI.

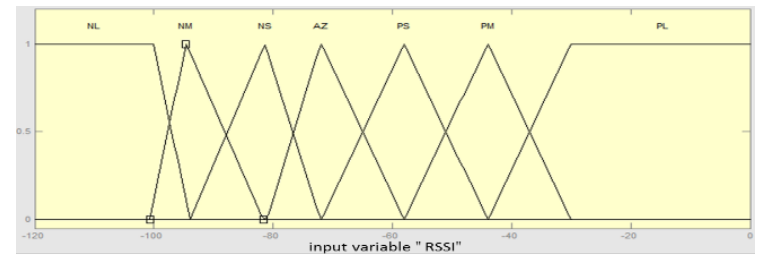

Fig. 5. Membership functions of RSSI.

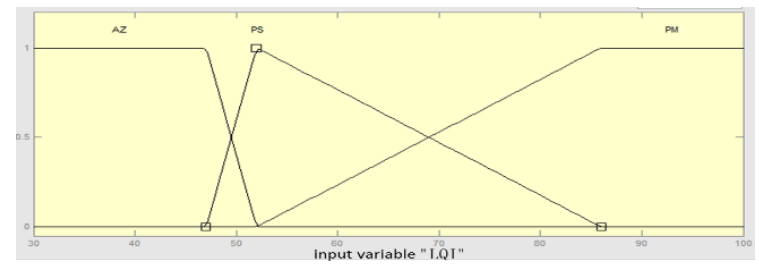

Fig. 6. Membership functions of LQI.

efficiency of the battery is of no significant improvement. 


\section{Architecture of Time Division}

Time division communication is technique of preventing package collision in communication by shifting the transmission time at which each node transmitting the package. The architecture of time division of this paper is shown in Fig. 9. $T_{\text {transform }}$ is the time of a node changing status from sleep to data transmission. $T_{\text {waiting }}$ is the waiting time which is the sum the time of sensing neighboring sensors around the nodes and $T_{\text {transform }}$. $T_{\text {get } N}$ is the data getting time for Node $N . T_{a c q}$ is the acquisition time of receiving information from all nodes.

The experimental results with battery of $1000 \mathrm{mAh}$ are summarized in Table 1. From Table 1, whether in shortdistance or long-distance, the designed TPC controller can make PER less than $1 \%$. If coupled with TDMA, the battery life can be increased by about 10 times.

Table 1. Experimental results of TDMA

\begin{tabular}{|c|c|r|c|c|}
\hline N-B dist. & Type & \#-Pack. & \#-Err-Pack. & PER (\%) \\
\hline \multirow{4}{*}{$1 \mathrm{M}$} & Normal & 237072 & 32 & 0.013 \\
\cline { 2 - 5 } & TPC & 237476 & 56 & 0.024 \\
\cline { 2 - 5 } & TDMA & 2030361 & 185 & 0.009 \\
\cline { 2 - 5 } & TDMA+TPC & 2030361 & 311 & 0.015 \\
\hline \multirow{4}{*}{$12 \mathrm{M}$} & Normal & 235292 & 8651 & 3.677 \\
\cline { 2 - 5 } & TPC & 234662 & 2087 & 0.889 \\
\cline { 2 - 5 } & TDMA & 2183322 & 52236 & 2.393 \\
\cline { 2 - 5 } & TDMA+TPC & 1977369 & 11172 & 0.565 \\
\hline
\end{tabular}

\section{Conclusion}

We have successfully designed an energy-saving architecture of transmission power control for wireless sensor network. Battery life is important for a wireless remote sensing node. With our design, the battery life can be extend to about 10 times and also the reliability of the data transmission can be improved. It is suitable for smart home or home-security system design.

\section{Acknowledgment:}

This work is partially supported by the Ministry of Science and Technology, ROC, under contract No. MOST 105-2221-E-224-024.

\section{References}

1. International Electrotechnical Commission, Internet of Things: Wireless Sensor Networks (International Electrotechnical Commission, Switzerland, 2014).

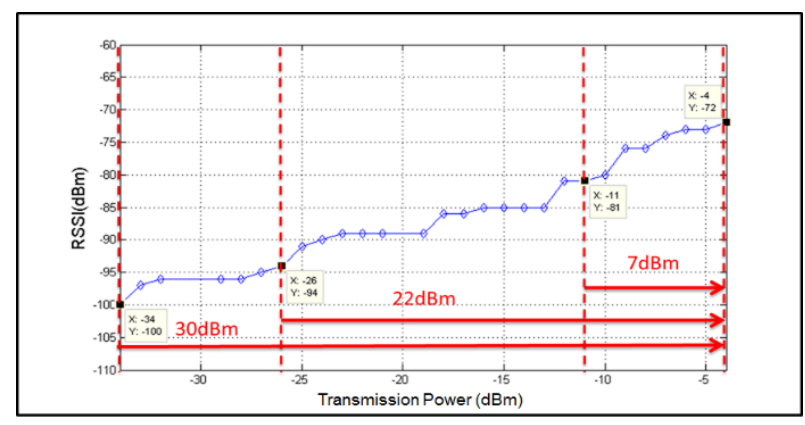

Fig. 7. Intervals of transmission power.

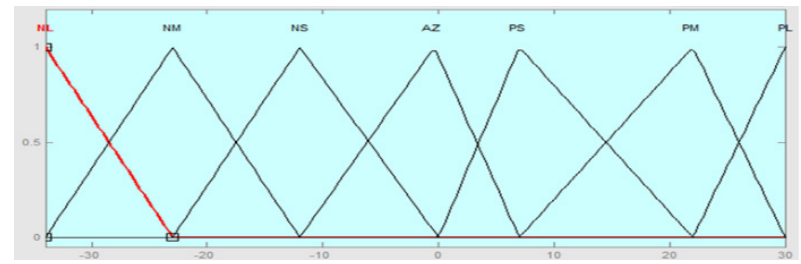

Fig. 8. Membership functions of PAP.

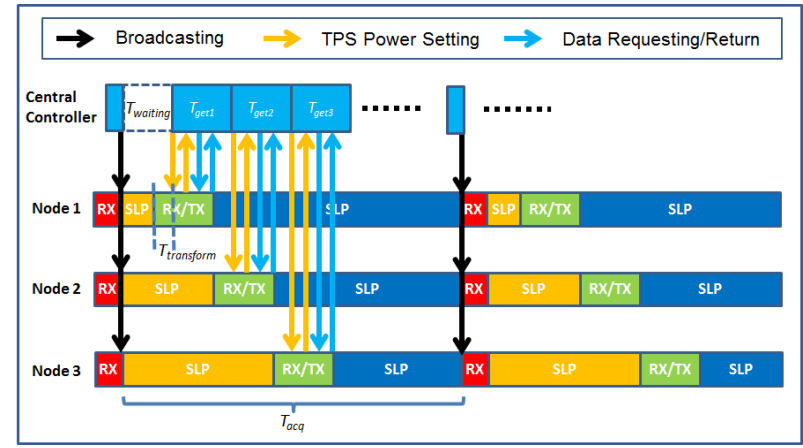

Fig. 9. Time division architecture.

2. W. Ikram, S. Petersen, P. Orten and N. F. Thornhill, Adaptive multi-channel transmission power control for industrial wireless instrumentation, IEEE Trans. on Industrial Informatics, 10(2) (2014) 978-990.

3. S. Ramakrishnan and B. T. Krishna, Closed loop fuzzy logic based transmission power control for energy efficiency in wireless sensor networks, in Proc. 2014 Int. Conf. Computer Communication and Systems, (IEEE, 2014), pp.195-200.

4. S. K. Kim and S. H. Kim, Doo-Seop Eom, RSSI/LQIbased transmission power control for body area networks in healthcare environment, IEEE J. Biomedical and Health Informatics, 17(3) (2013) 561-571.

5. S. Thodupunoori, Reverse Link Performance of an $1 x E V$ DO Revision A System, Master Thesis (University of Missouri-Kansas City, 2008).

6. S. Myneni, Application Note -- MRF89XA Radio Utility Driver Program (Microchip Technology Inc., 2010).

7. M. Petrova, J. Riihijarvi, P. Mähönen and S. Labella, Performance Study of IEEE 802.15.4 Using Measurements and Simulations, in Proc. 2006 IEEE Wireless Communications and Networking Conf. WCNC2006 (IEEE, 2006), pp. 487 - 492 